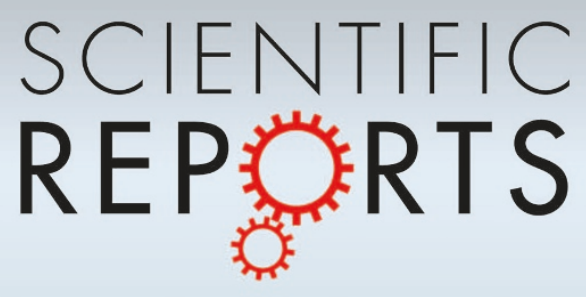

OPEN

SUBJECT AREAS:

COMPUTATIONAL

BIOLOGY AND

BIOINFORMATICS

COMPUTATIONAL BIOPHYSICS

MEMBRANE BIOPHYSICS

Received

8 September 2014

Accepted

24 November 2014

Published

12 December 2014

Correspondence and requests for materials should be addressed to

A.O.C. (batch2k@ yandex.ru)

\section{Liquid but Durable: Molecular Dynamics Simulations Explain the Unique Properties of Archaeal-Like Membranes}

\author{
Anton O. Chugunov' ${ }^{1}$ Pavel E. Volynsky ${ }^{1}$, Nikolay A. Krylov ${ }^{1,2}$, Ivan A. Boldyrev' \& Roman G. Efremov 13,4
}

'M.M. Shemyakin \& Yu.A. Ovchinnikov Institute of Bioorganic Chemistry, Russian Academy of Sciences, ul. Miklukho-Maklaya, 16/ 10, Moscow 1 17997, ${ }^{2}$ Joint Supercomputer Center, Russian Academy of Sciences, Leninsky prospect, 32a, Moscow 119991 , Russia, ${ }^{3}$ Moscow Institute of Physics and Technology (State University), Dolgoprudny, Moscow Region, 141700, Russia, ${ }^{4} \mathrm{Higher}$ School of Economics, Myasnitskaya ul. 20, 101000 Moscow, Russia.

Archaeal plasma membranes appear to be extremely durable and almost impermeable to water and ions, in contrast to the membranes of Bacteria and Eucaryota. Additionally, they remain liquid within a temperature range of $0-100^{\circ} \mathrm{C}$. These are the properties that have most likely determined the evolutionary fate of Archaea, and it may be possible for bionanotechnology to adopt these from nature. In this work, we use molecular dynamics simulations to assess at the atomistic level the structure and dynamics of a series of model archaeal membranes with lipids that have tetraether chemical nature and "branched" hydrophobic tails. We conclude that the branched structure defines dense packing and low water permeability of archaeal-like membranes, while at the same time ensuring a liquid-crystalline state, which is vital for living cells. This makes tetraether lipid systems promising in bionanotechnology and material science, namely for design of new and unique membrane nanosystems.

A

rchaea are microorganisms that thrive predominantly in extreme conditions (very high/low temperatures, high pressure, acidity and/or salinity), although many of them inhabit more "common" econiches (ocean water, soil, intestine, etc) ${ }^{1}$. According to pioneering works by Carl Woese, Archaea are a group apart not only ecologically, but also evolutionary ${ }^{2,3}$, nowadays designated as the third domain of life (along with Bacteria and Eucaryota). One hypothesis suggests that the separation of Bacteria and Archaea resulted from the individualization of membranes, which were "promiscuous" in the Last Universal Common Ancestor (LUCA) and became more specialized in the two modern domains of microorganisms due to the so-called "Lipid Divide" ancient event ${ }^{4}$. There are two major differences in membrane lipids structure between Archaea and Bacteria: 1) glycerol backbone chirality and the way the hydrophobic "tails" are connected, and 2) the chemical nature of these tails. In Bacteria and Eucaryota, straight fatty acid acyl chains are linked by ester bonds to sn- 1 and sn- 2 positions of glycerol, while in Archaea, branched isoprenoid hydrocarbon chains are bound by ether bonds to sn- 2 and sn-3 glycerol positions ${ }^{5}$. Glycerol backbones are enantiomers in both Bacteria and Archaea. The polar heads used are also often different, although they are not unique to any domain of life.

Of the two aforementioned chemical differences between bacterial and archaeal lipids, glycerol chirality may have played a very important evolutionary role ${ }^{4,6}$, although it most probably does not affect the physical properties of the membranes. In contrast, the primordially branched isoprenoid chains in Archaea are likely to determine the very dense packing ${ }^{7,8}$, high membrane viscosity ${ }^{9}$ and durability ${ }^{10}$ and low permeability to water and ions, which are the main characteristics of archaeal membranes compared with bacterial or eukaryotic ones ${ }^{11-13}$. An additional modification that increases the density of packing and decreases membrane permeability is cyclopentane rings in the hydrophobic tails, which are encountered more often in acidophilic microorganisms $\mathrm{s}^{7,14}$. Moreover, Archaea are able to adapt their membranes to better fit their growth conditions ${ }^{5,15}$, employing several types of lipids' structure variation. Finally, most hyperthermophiles contain lengthy C40-based glycerol-dialkylglyceroltetraether (GDGT) lipids, which are usually referred to as caldarchaeols or bolalipids ${ }^{16}$ and represent "tail-to-tail" linked glycerol-dialkyl-glyceroldiether (GDGD) lipids, or archaeols. This modification provides extra durability for the membranes by switching from a bilayer to a more rigid monolayer structure.

In spite of these extraordinary mechanical properties reminiscent of a gel state of "conventional" dipalmitoylphosphatidylcholine (DPPC) ${ }^{8}$, archaeal membranes may be considered liquid crystalline within a very broad temperature range $\left(0-100+{ }^{\circ} \mathrm{C}\right)^{17,18}$, which is believed to be vital for each living cell. At the same time, very high 
durability and liquidity seem to be the most fundamental physical properties of archaeal membranes. This not only provides an insight into the evolutionary fate of Archaea, but may also be adopted by bionanotechnology in order to design new unique materials ${ }^{19}$. In order to achieve this, a detailed understanding of the physico-chemical properties of such membranes is required. In contrast to "common" bilayer membranes, archaeal membranes are monolayers, and the organization of their nonpolar core can strongly depend on the occurrence of various branching groups in the tetraether lipids. Experimental investigations in this field are hindered by the complex mixture of lipid extracts from archaeal membranes and the complexity of the chemical synthesis of the GDGT lipids. Hence, computer experiments may help provide relevant information with regards to the microscopic organization of such membranes.

Although this task is within reach of modern computational methods, only a few research papers have been published in this field. The most important work is by Bulacu et al. ${ }^{20}$, who performed a molecular dynamics (MD) simulation of a bolalipid membrane in order to find out the effect of lipids' tail linkage. However, due to the coarsegrained representation used, the role of branching methyl and cyclopentane groups could hardly be assessed, although the authors provide some insights into the phase condition and design of synthetic durable membranes. In their early work, Gabriel \& Chong ${ }^{21}$ assessed via MD the role of cyclopentane rings in the tight packing of the membrane, although they did not systematically address the influence of branched methyl groups. Another work has shown the effect of tail linkage in archaeal lipids: expectedly, tetraether lipids exhibit lower lateral diffusion compared with diether ones ${ }^{22}$.

The second issue to be carefully considered is the juxtaposition of the atomistic-scale properties of membranes composed of tetraether and "conventional" lipids. Apart from its fundamental importance, the task seems to be very promising from the point of view of developing new nanomaterials, e.g., lipid films combining the properties of biological membranes with the durability of artificial ones. It is well established now that biomembranes have a highly heterogeneous and fine-tuned organization, which ensures their adaptability to external agents - peptides, proteins, and so on ${ }^{23-25}$. This is largely determined by the bilayer organization of bacterial and eukaryotic membranes. Hence, mixing them with archaeal lipids may result in membranes with unprecedented properties but still capable of accomplishing their biological function.

In this work, we examine the effect of a model bolalipids chemical structure on the physical properties of membranes composed of these molecules. Using MD simulations of five bolalipid mimetics, we conclude that the branching of hydrophobic tails is fundamental to the unique physicochemical properties of archaeal membranes, which are extremely durable (e.g., stable under extreme physical conditions) and liquid at the same time. We then provide an extensive comparison of the spatial structure, dynamics and other parameters of these systems with those observed for "standard" lipid bilayers. The common and specific characteristics of these membranes are discussed.

\section{Results and Discussion}

Choice of bolalipid mimetics. To assess the effect of branching groups of bolalipids' hydrophobic tails on the structural, dynamic and hydrophobic organization of the model archaeal membranes in a systematic manner, we simulated several hydrated monolayers composed of bolalipids, with hydrophobic tails gradually varying from straight C32 alkyl chains to archaeal-like C40 methyl-branched isoprenoid chains. The glycerol backbone stereochemistry and the way in which the hydrophobic tails are connected were archaeal-like, whereas polar heads were phosphatidylcholines (PC) - the most common variant in Eucaryota. PC was chosen in order to focus primarily on the effect of the chemical structure of the hydrophobic tails and compare the results with those obtained for common PC-based phospholipids - dipalmitoylphosphatidylcholine (DPPC) and palmitoyloleylphosphatidylcholine (POPC). Five mimetics of bolalipids were selected for the computational analysis. They differed by the number and chemical nature of the branching groups: methyls $(\mathrm{m})$ and cyclopentane rings ( $\mathrm{r}$ ). The "core" mimetic does not contain any modifications and hereinafter is referred to as m0r0 (this one is the most "artificial"). M8r0 and m6r2 mimetics have eight branching modifications in each tail (eight methyl groups and six methyl groups plus two rings, respectively) (these two are the most "native-like"). Besides these, two "intermediate" mimetics, m0r2 and m2r0, which contain two branching modifications per tail (two rings or two methyls, respectively), were chosen. The chemical structure of all five mimetics (along with DPPC and POPC) is shown in Fig. 1.

Flowchart of the study. 1) Assembly of hydrated monolayer membranes from bolalipid mimetics and calculation of molecular dynamics (MD). 2) Assessment of the packing characteristics describing the conformational properties of bolalipid molecules in the model membranes. 3) Characterization of phase condition of the model membranes depending on the computed "liquidity" (or "plasticity") of the hydrophobic core of the membrane and the ordering of lipid molecules. 4) Estimation of the permeability of the model membranes to water molecules. 5) Assessment of the fine packing details in the membranes: free volume, polar heads clustering, water-membrane hydrogen bonds. From these analyses, we draw conclusions about the physical properties of the model bolalipid membranes relative to "common" phospholipid bilayers. A similar procedure was also used for the DPPC and POPC bilayers. We describe each of these points in detail below.

Collection of MD data. For each of the five mimetics, the modeled molecules were arranged to form a $12 \times 12$ monolayer (see Fig. 2). The membranes were solvated with water, and MD was simulated at four different temperatures $(290,310,330$ and $350 \mathrm{~K})$ each. If not explicitly mentioned, all results hereinafter correspond to $350 \mathrm{~K}$ (higher temperature provides for a better exploration of conformational effects); a complete dataset is provided in Table S1 and Figs. S1-S3. Simulation details are provided in Computational methods.

Bolalipids packing in the model membranes. One of the important results of the simulations can be seen with the naked eye: the completely straight alkyl hydrophobic tails (without side methyl groups) in the m0r0 membrane, which are almost crystallographically ordered, strongly reminiscent of a gel phase, while the fully branched m8r0 membrane looks rather similar to common lipid bilayers (Fig. 2B,C). The lipid molecules in m0r0 appear to be extended, tilted with respect to the membrane normal and highly parallel to each other. To characterize this quantitatively, we introduce parameters of lipid "length" $(L)$, tilt angle $(\chi)$ and collinearity angle $(\varphi)$, which are explained in Fig. $2 \mathrm{~A}$ and in Computational methods. We study the MD-distributions of these parameters (Fig. 3) in order to formalize our initial evaluation.

An analysis of Fig. 3 provides evidence that the branching of the bolalipid tails plays an important role in the phase behavior of the model membranes, a role reminiscent of unsaturated bonds in bacterial and eukaryotic phospholipids. Membranes made of m8r0 and m6r2 mimetics that possess eight branching modifications in each tail form a partially disordered, flexible phase (we can call it "liquidcrystalline"). At the same time, m0r0, m0r2 and m2r0, which either do not have such modifications or have only two of them, form a rigid phase (which can be designated as gel-like). This phase is characterized by stretched hydrophobic tails (Fig. 2B,C; Fig. 3B), high lipid tilt angle $\chi$ (Fig. 3A), an almost complete collinearity of the molecules within the MD box (Figs. 2B and 3C), and a high-density packing of the hydrophobic core (Fig. 3D). Considering the native size of the phosphatidylcholine head of $0.7-0.8 \mathrm{~nm}$ and an "optimal" inter- 


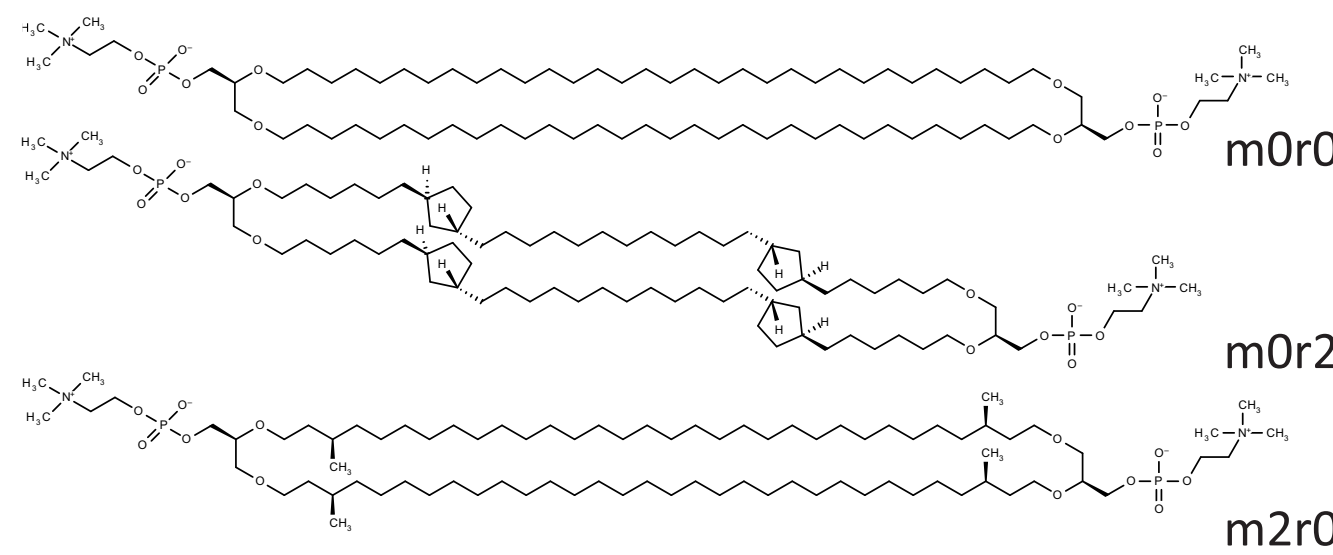

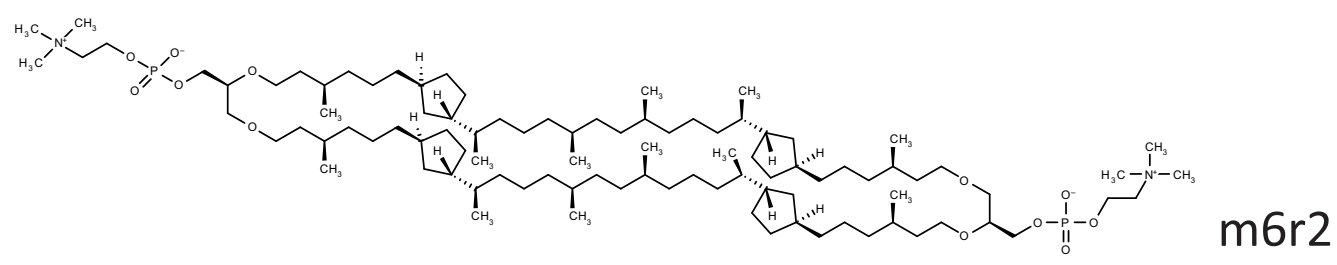

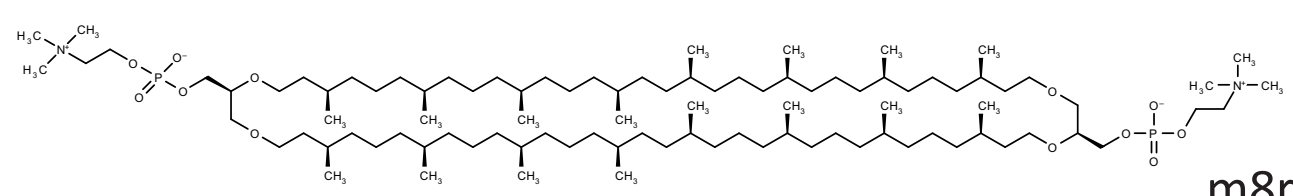

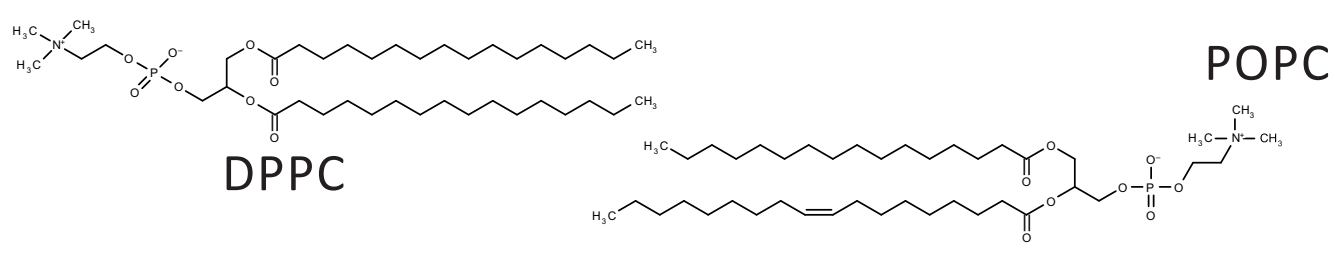

Figure $1 \mid$ Chemical structures of lipid molecules in this MD-study. Five upper molecules are mimetics of archaeal bolalipids. The "core" structure represented by the m0r0 molecule consists of two straight O-linked C32 alkyl chains, connected to an archaeal-type glycerol molecule with ether bonds. In all cases, the hydrophilic heads are phosphatidylcholines. Other mimetics (m0r2, m2r0, m6r2 and m8r0) have different numbers of branching modifications of the hydrophobic core: methyl groups (designated as " $m$ " in mimetic codes) and cyclopentane rings (designated as " $r$ "). The lower row contains dipalmitoylphosphatidylcholine (DPPC) and palmitoyloleylphosphatidylcholine (POPC), which were chosen for comparison.

chain distance of $\approx 0.6 \mathrm{~nm}$ for "straight" tails in the m0r0 mimetic, the tilt of the whole chains $\left(\approx 35^{\circ}\right)$ may be driven by this factor. The introduction of side methyl groups (characteristic size $\approx 0.25 \mathrm{~nm}$ ) increases the interchain distance to $\approx 0.85 \mathrm{~nm}$, which in turn decreases the average tilt angle to $\approx 10^{\circ}$. Therefore, the packing geometry of bolalipid layers may be varied depending on the choice of polar heads. These results hold for all the temperatures examined; the full set of distributions and MD-calculated parameters is provided in Table S1 and Figs. S1-S3. A more detailed analysis of phase conditions in the model membranes is provided in the next section.

The partial density profiles in Fig. 3D show a number of sharp peaks, which correspond to the cyclopentane rings. This further improves the packing (partial density increases from 870 to $970 \mathrm{~kg} / \mathrm{m}^{3}$ in the case $\mathrm{m} 8 \mathrm{r} 0 / \mathrm{m} 6 \mathrm{r} 2$ mimetics pair) and decreases the
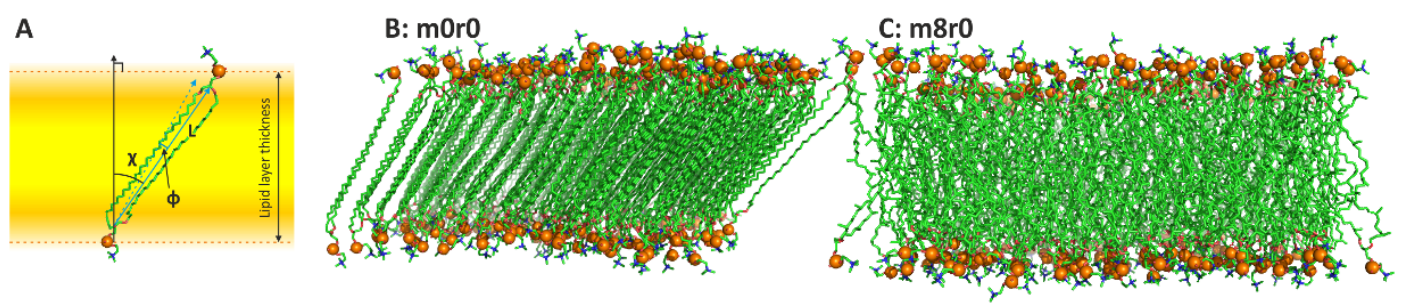

Figure $2 \mid$ Model bolalipid membranes. (A). Position of an individual bolalipid molecule with respect to the model membrane (schematically depicted with the yellow layer). Phosphorus atoms are shown with orange spheres; they define the thickness of the membrane. For the purposes of this analysis, each lipid is represented with a vector (blue arrow) built with two sn-2 carbon atoms from opposite glycerol backbones. The length of this vector ( $L$ ) characterizes the conformation of the lipid (extended/expanded). The lipid tilt angle $(\chi)$ is measured with respect to the membrane normal (black arrow). The lipid-lipid "collinearity" angle $(\varphi)$ is measured between each pair of vectors within one MD snapshot (the second vector is shown with a blue broken arrow). (B). Model membrane of the m0r0 bolalipid mimetic (with completely "straight" tails, without methyl side groups) after MD simulation. (C). Model membrane of the m8r0 mimetic, which contains a branched methyl group in every fourth position in the hydrophobic tail. 

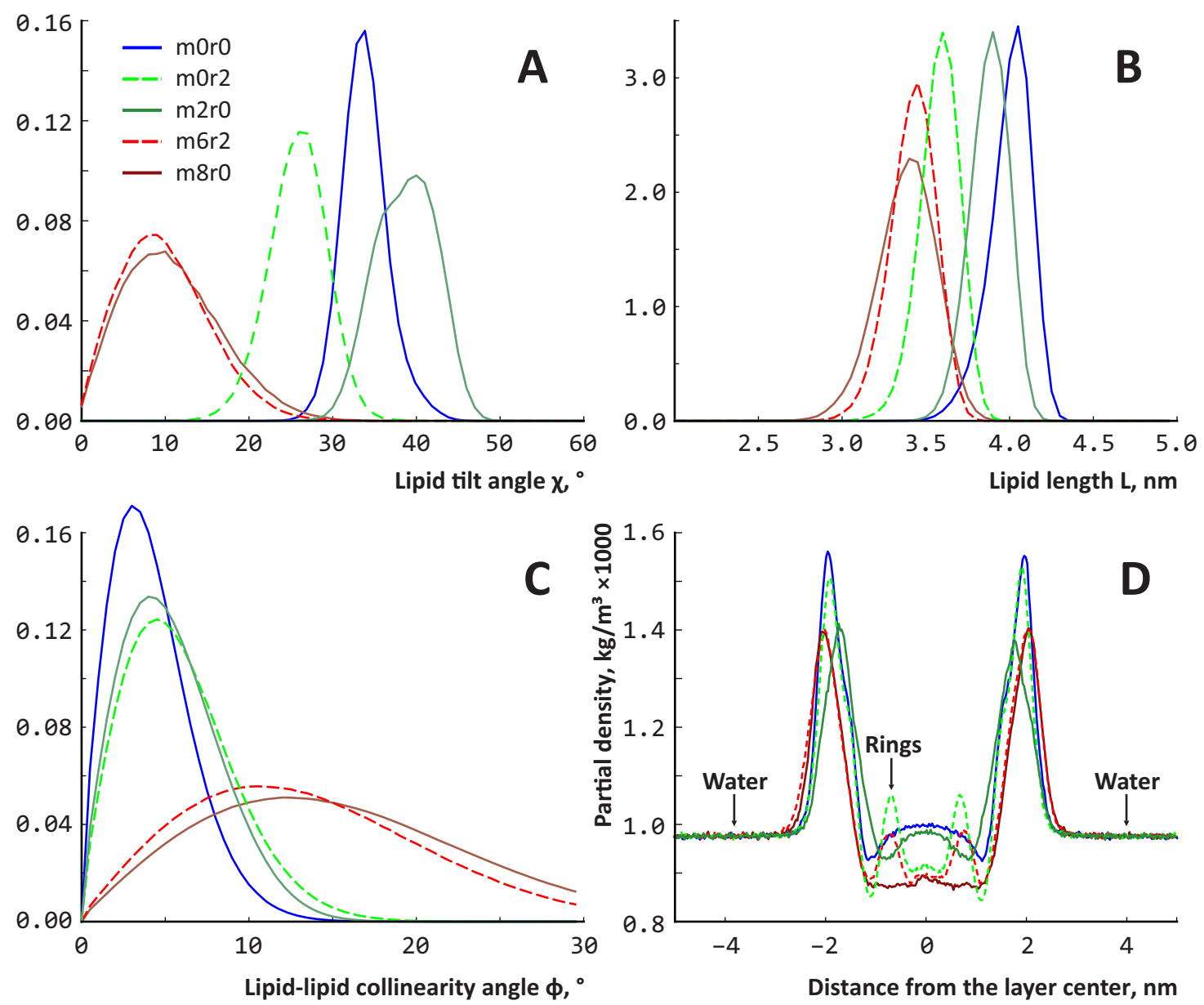

Figure 3 Packing parameters in the model bolalipid membranes. Each bolalipid mimetic is represented by a different color and line style, as shown in the legend. Panels A-C depict the probability density functions. All parameters are provided for $350 \mathrm{~K}$ MD runs. Supplementary Table S1 and Figs. S1-S3 contain the calculated parameters and distributions, respectively, for the whole temperature range examined. (A). Lipid tilt angle with respect to the layer normal $(\chi)$. Note that $\mathrm{m} 8 \mathrm{r} 0$ and $\mathrm{m} 6 \mathrm{r} 2$ are less tilted. (B). Lipid “length" $(L)$, which describes the molecules' conformation inside the membrane. Note that $\mathrm{m} 8 \mathrm{r} 0$ and $\mathrm{m} 6 \mathrm{r} 2$ have the smallest length (e.g., molecules are expanded), while m0r0 has the greatest (molecules are extended). (C). Lipid-lipid "collinearity angle" $(\varphi)$ between all the molecule pairs within one MD snapshot. Note that molecules in m8r0 and m6r2 are less collinear and more independent from each other. (D). Partial density profiles of the whole systems (including solvent and lipid molecules) along the membrane normal. Note that in the center of the membrane, the least densely packed are $\mathrm{m} 8 \mathrm{r} 0$ and $\mathrm{m} 6 \mathrm{r} 2$, while $\mathrm{m} 0 \mathrm{r} 0$ is the densest. The m0r2 and m6r2 profiles exhibit peaks corresponding to cyclopentane rings (shown with an arrow).

membrane's permeability for the solvent (up to 10 times, see below), which is vital for survival in extreme conditions. It has been shown that Archaea species that reside in low-pH and high-salinity conditions have more cyclopentane rings in their lipids' tails, and this number varies depending on growth conditions. In fact, the maximum ring number is achieved in conditions which are optimal for this species.

Unusual phase condition of bolalipid membranes. One of the hallmarks of archaeal membranes is the lack of distinct phase transitions and relatively weak dependence of the membrane's properties on temperature. This implies an unusual phase condition of the bolalipid membranes, which are very densely packed but liquid at the same time. In order to better characterize this phase condition computationally, we used two approaches. The first one estimates the "liquidity" of the hydrophobic core of the membrane; the second is aimed at detecting the long-range ordering of lipid molecules in the membrane as a distinct feature of the rigid "gel" phase (2D crystal).

The "liquidity" of the membrane, which is a requirement for its biological function, is assessed with the Root-Mean-Square Fluctuation (RMSF) parameter, which was calculated for bolalipids' hydrophobic tails as a function of the carbon atom number (see Fig. $4 \mathrm{~A}$ and Computational Methods). (More common parameter - lateral diffusion coefficient - is not applicable here, since in most systems bolalipids cannot diffuse freely and their motion cannot be described by this quantity - at least at the time scales achieved in MD simulations.) The RMSF gradually increases as the number of branching modifications in the hydrophobic tail rises from $\approx 0.08 \mathrm{~nm}$ for $\mathrm{m} 0 \mathrm{r} 0$ to $\approx 0.22 \mathrm{~nm}$ for m8r0. Importantly, the highly branched $\mathrm{m} 8 \mathrm{r} 0$ appears to be more "liquid" than the straight m0r0. Moreover, temperature dependence, which reveals a phase transition in DPPC (Fig. 4B; kink in the black line), shows a uniform RMSF increase for each bolalipid mimetic, suggesting the absence of a phase transition in the bolalipids. Interestingly, m8r0 has the highest slope and therefore the most rapid increase of "liquidity" compared with the other mimetics, nearly reaching the level of POPC and DPPC at elevated temperatures, while being substantially more rigid at, say, $310 \mathrm{~K}$. This provides evidence that the branching of hydrophobic tails gives the bolalipid membranes their characteristic properties, namely high durability and, at the same time, liquidity. (In this work, we call "durable" the membranes that are resistant to physical destruction upon extreme conditions, and "liquid" those having flexible and fast-fluctuating tails in the hydrophobic core.) 

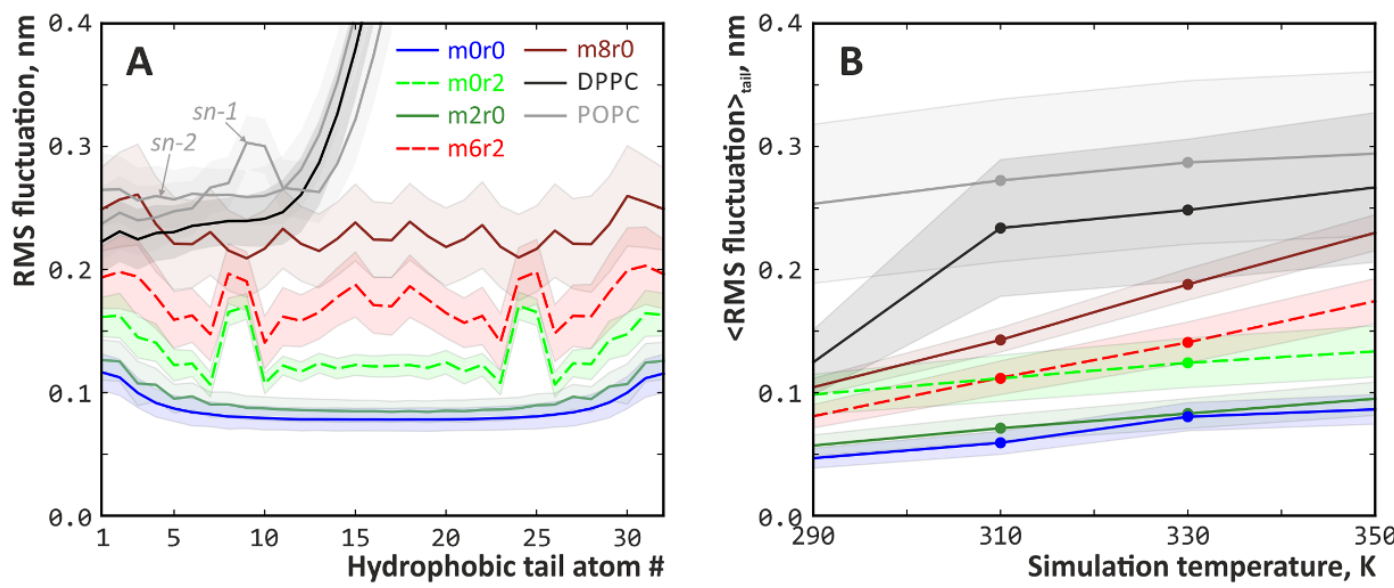

Figure 4 Bolalipid mimetics with branched tails are more "liquid" than straight-tailed ones. The measure of "liquidity" is the MD-averaged RMS fluctuation (RMSF) of either a certain tail atom $(A)$ or the whole tail $(B)$ (see Methods). (A). RMSF profiles for five bolalipid mimetics, DPPC, and POPC (see legend). Shaded areas represent standard deviation. Simulation temperature is $350 \mathrm{~K}$. (B). Temperature dependence of tail-averaged RMSF $(<$ RMSF $>)$.

The long-range ordering of molecules is a hallmark of a crystal phase in general and a gel phase in particular for membrane lipids. We used radial distribution functions (RDFs) to uncover such an ordering in the model membranes. Several distinct peaks in the RDF point to a gel phase in DPPC at low temperatures (290 K; see Fig. S4B); at the same time, the liquid crystalline phase exhibits only one or two diffuse peaks, which are characteristic of POPC for all temperatures examined (Fig. S4A). A similar analysis for the bolalipid mimetics (Fig. S4C) shows distinct peaks for the m0r0, $\mathrm{m} 2 \mathrm{r} 0$ and m0r2 systems (indicating a gel-like phase), while m6r2 and m8r0 have more diffuse RDFs (indicating a liquid-crystalline phase).

Both the "liquidity" and "ordering" analyses confirm that hydrophobic tail branching modifications render membranes more liquid and less rigid, which is a requirement for the membranes' biological function. Still, packing remains dense, making the membrane durable. Additionally, all the calculated parameters change only slightly as temperature increases (see Table S1 and Figs. S1-S3). For example, m0r0 remains in a gel-like phase at all temperatures, whereas m6r2/ $\mathrm{m} 8 \mathrm{r} 0$ are in a liquid-crystalline state within the whole temperature range examined. This feature may be an important prerequisite for Archaea to thrive in a wide range of econiches, from cold ocean water to high-temperature and pressure hydrothermal vents.

Water permeability across the bolalipid membranes. Long-term MD trajectories allow us to assess the water permeability of the model membranes (see Computational Methods). Figure 5 shows the frequency of water permeation events (due to free diffusion) across the five model bolalipid membranes, and also across two "common" phospholipid bilayers for comparison purposes. The gel-like m0r0 membrane was impermeable to water (not a single molecule passed across the layer throughout the whole observed time interval, $100 \mathrm{~ns})$. M6r2 and m8r0, which are considered to be in a liquidcrystalline state, let through approximately 15 water molecules per 100 ns of analyzed trajectory, $\approx 5$ times fewer than phospholipid POPC bilayer. The m0r2 and $\mathrm{m} 2 \mathrm{r} 0$ systems, which possess only two branching modifications in their hydrophobic tails, have very small, albeit non-zero permeability. There is no significant difference in water permeability between membranes with and without cyclopentane rings (compare m0r2 vs. $\mathrm{m} 2 \mathrm{r} 0$ and $\mathrm{m} 6 \mathrm{r} 2$ vs. $\mathrm{m} 8 \mathrm{r} 0$ ). However, an important difference may exist for charged particles (protons and other ions), which was not assessed in this work.

Free volume inside the bolalipid membranes. In MD trajectories, we have observed water molecules that spontaneously permeate most of the membranes under study. This process seems stochastic, nearly always involving single water molecules, and not exhibiting formation of any kind of the pore of H-bonding networks of waters in the central part of the membranes. The permeation of water across hydrophobic layers requires the formation of dynamic (semi)continuous void spaces between the lipids' hydrophobic tails ${ }^{26}$. To uncover the structure and characteristics of the voids inside the various membranes, we have developed an algorithm to "map" such voids in three dimensions (see Computational methods). This allows us to plot the profiles of free volume depending on the $\mathrm{Z}$ coordinate in the membrane ( $\mathrm{Z}$ is the distance from the membrane's middle plane). Fig. 6 depicts these profiles for all the examined systems at $350 \mathrm{~K}$. Three-dimensional picture of these voids is provided in fig. S6. The main observation is free volume peaks in the bilayer center for phospholipids; additionally, the corresponding voids are fluctuating fast. However, this is a direct consequence of the chemical structure: lipids' tails from opposite leaflets are not chemically linked. At the same time, the water-membrane interface is organized similarly in phospholipids and branched bolalipid mimetics m8r0 and $\mathrm{m} 6 \mathrm{r} 2$.

An analysis of the bolalipids' profiles shows that the highlybranched bolalipids $\mathrm{m} 6 \mathrm{r} 2$ and $\mathrm{m} 8 \mathrm{r} 0$ have non-zero free volume in the membrane center, and the ring-containing m0r2 and m6r2 have peaks of free volume just "above" the layer containing cyclopentane rings. Straight or almost straight tails in m0r0 and $\mathrm{m} 2 \mathrm{r} 0$ do not leave voids in between, making the membrane core almost impermeable to water in these systems. All in all, the tails of the highly-branched bolalipids leave considerably less free space in the membrane com-

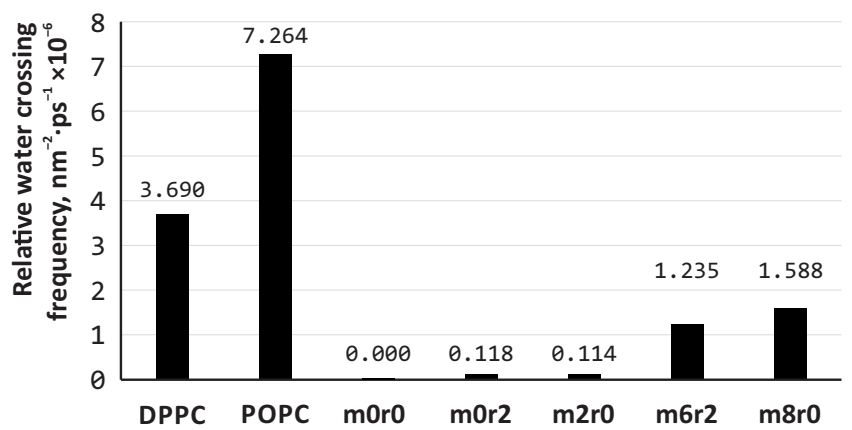

Figure $5 \mid$ Permeation of water across the membranes. The measured quantity is the event frequency of water passing through the membrane. Simulation temperature is $350 \mathrm{~K}$; values for other temperatures are listed in Table S1. 
pared with phospholipids, although the typical void lifetimes are longer. This peculiar interplay between the structure and dynamics of the cavities inside the membrane core still creates an opportunity for water molecules to diffuse across the membrane (Fig. 5), apart from straight tail bolalipids, which appear almost impermeable.

Analysis of single permeation events reveals three stages of water molecule passing across the membrane: 1) approach to the membrane surface; 2) rapid "fall" to the membrane center with optional "hanging" there for a period of time, and 3) leaving the membrane (fig. S7). Interestingly, POPC phospholipid and m8r0 bolalipid exhibit similar "fast" jumps with minimal time of water molecule passing across the membrane of $\approx 50$ ps (Fig. S7A), but distinct "long" jumps, which are up to $\approx 500-750$ ps for POPC and up to 1800 ps for m8r0 membrane (Fig. S7B). (We should note that parameters of spontaneous water diffusion in other lipid bilayers are rather similar to those in POPC (see ref. 26 for more details).)

Analysis of the water-membrane interface. In our bolalipid mimetics, we use common PC polar heads. At first glance, this implies surface properties similar to those in phospholipid membranes. Indeed, many important parameters, such as area per lipid, membrane thickness (Table S1), free volume in the interface area (Figs. 6 and S5), and number of hydrogen bonds per lipid head (Fig. S9B) are very similar. In other words, despite the dramatic differences in the organization of the nonpolar membrane core, PCs serve as a specific "buffer" between the membrane interface and the free solution. Previously, such a decoupling of interfacial and bulk water properties was observed in experiments ${ }^{27}$ and simulations ${ }^{26}$. At the same time, an intent look at bolalipid systems' interfaces reveals that the aforementioned similarity with lipid bilayers is valid only for the integral parameters of the membranes. Apart from them, there are several important differences in the local organization of bolalipid and phospholipid membranes. They are related to the water dynamics and clustering of lipids in the interfacial zone.

Firstly, water molecules slow down considerably in the watermembrane interface compared with bulk solution (Fig. S8), and they do this rather more efficiently in bolalipid than in lipid membranes. The most significant retardation is observed for the "rigid" m0r0 (Fig. S8). In order to delineate possible reasons for this observations, we analyzed parameters of water $\mathrm{H}$-bonding along the membrane normal. The average lifetime of hydrogen bonds between water

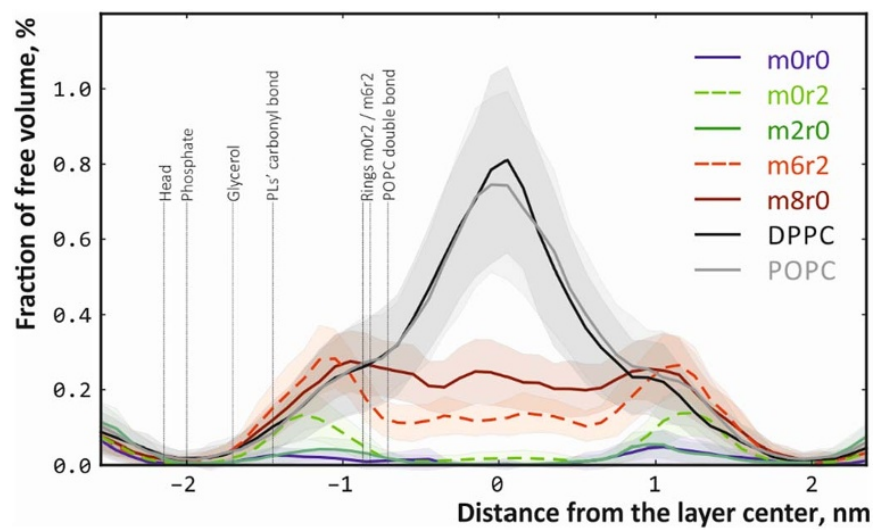

Figure 6 | Free volume inside the membranes. The fraction of free volume is given as a percentage with respect to the total volume of the membrane slice, depending on the $\mathrm{Z}$ coordinate of the slice (for details of the calculation, see Computational methods). Five free volume profiles are presented for the bolalipid systems and two profiles for the phospholipid systems, colored and styled according to the legend. Shaded areas are standard deviations. This series of profiles was obtained at $350 \mathrm{~K}$. Vertical lines show the average positions of lipids' chemical groups (choline head, phosphate, glycerol, etc.). An analogous plot for $310 \mathrm{~K}$ is shown in Fig. S5. molecules and lipid head groups increases, reaching a maximum of 80 ps for m0r0, compared with 20 ps for the POPC membrane (Fig. S9A). At the same time, the average number of h-bonds per lipid head group remains constant (Fig. S9B). It is noteworthy that even in the highly-branched bolalipid membrane (m8r0), an apparent close analog of lipid bilayers, the dynamics of PC-water hydrogen bonds is much slower ( $\approx 45 \mathrm{ps})$ than in POPC and DPPC ( $<30 \mathrm{ps}$ ) (Fig. S9).

Secondly, the lateral clustering of $\mathrm{PC}$ heads in both types of membranes (bolalipids $v s$. lipids) is different as well. Thus, despite rather dissimilar physico-chemical properties ${ }^{24}$, the mean fraction of clustered lipids in the bilayers is constant - about 25-30\% (Fig. S10). In addition, the size distributions of clusters are almost the same (data not shown). In contrast, different bolalipids exhibit a much more heterogeneous degree of clusterization - from $13 \%$ in $\mathrm{m} 8 \mathrm{r} 0$ to $50 \%$ in m0r0 (Fig. S10). Moreover, the corresponding size distributions of the clusters vary considerably - the smallest ones are observed in m8r0 (data not shown). Therefore, despite their structural "rigidity" (both in the core hydrophobic part and the interface), bolalipid membranes regulate their local interfacial properties within a wider range than lipids - depending on the tails' branching groups.

This provides additional support to the picture of a fast-changing dynamic water-membrane interface in phospholipid as well as highly-branched bolalipid membranes, in contrast to the rigid and mostly static organization of the m0r0 membrane. From this point of view, the straight-tailed m0r0 mimetic has the densest packing with highly-clustered lipid chains and very little free volume between its hydrophobic tails. The $\mathrm{PC}$ heads in $\mathrm{m} 0 \mathrm{r} 0$ are also static and represent a rigid and rather regular "matrix" able to decelerate and capture water molecules. At the same time, the highly-branched m8r0 mimetic is much more dynamic and loosely packed with a higher amount of free space inside the membrane.

Taken together, our simulation results show that the properties of archaeal-like membranes are determined by a combination of finely regulated macroscopic and microscopic structural and dynamic factors. Despite being substantially less subjected to random fluctuations than lipid bilayers, bolalipid systems still manifest a number of small but fundamental effects, which locally control the behavior of their membranes. Such phenomena can be "trapped" only in atomistic simulations. They cannot be correctly described solely within the framework of continuum models, even if they perfectly reproduce the available macroscopic experimental data. This proves the utility and wide perspectives of computational approaches in understanding the molecular details of the organization and biological functioning of Archaeal membranes.

\section{Conclusions}

Our MD results provide evidence that the unique high durability and low permeability of archaeal membranes, along with their liquidcrystalline state within a very broad temperature range, are determined by the chemical nature of isoprenoid tails themselves along with the branching groups, which are mostly side methyls and chainincorporated cyclopentane rings. Apart from phospholipid bilayers, the low amount of fast-fluctuating void spaces inside the membrane ensures low permeability of the bolalipid layers for the solvent and the rather unique microscopic organization of their interface - even with the PC polar heads inherent in convenient lipid membranes.

A thorough comparison of the atomistic-scale properties of archaeal-like membranes and "common" lipid bilayers reveals a very intriguing picture. Namely, due to the free ends of acyl chains, the hydrophobic core of lipid bilayers is rather dynamic and characterized by fast large-scale fluctuations, clustering and appearance of free volumes $^{24,28}$. The bilayer interface is dynamic as well (although to a lesser extent) and manifests a mosaic nature, mainly determined by the type of polar heads ${ }^{24}$. Such complex behavior is biologically relevant because it ensures a fast and adequate reaction of bacterial and eukaryotic membranes to a wide variety of scenarios of functioning, 
including adaptation to binding, insertion of peptides and proteins, membrane fusion and rearrangements, and so on. Despite these local perturbations, lipid bilayer membranes preserve their interfacial organization from external intrusions well. Water plays a crucial role in this ${ }^{26,27}$. As we demonstrate here for the first time, the analogous characteristics of archaeal-like membranes exhibit a prominent dualism - while some of them very closely resemble lipid bilayers, others are drastically different. For instance, the tight packing of lipid chains prevents the appearance of voids and fluctuations in the hydrophobic domain, although the mosaic nature and water dynamics in the interface can be efficiently regulated within a wide range, namely by the incorporation of different branching groups into the lipid tails. This allows for the creation of membranes with unique mechanical properties (durability, stability at high temperatures/pressure, etc.), but still biologically active. It is noteworthy that the response of bolalipids to temperature changes can also be very unusual, unlike in lipid membranes. These peculiarities of atomistic structure of the hydrophobic core of the membrane may have provided Archaea with the ability to thrive in the harshest environments.

The extraordinary ability of bolalipid membranes to vary their physical properties depending on the number and chemical nature of side branching groups sheds some light on the next steps in the rational design of novel materials for biotechnology and medicine, based on archaeal-type lipids. Future theoretical works should pay attention on modeling of mechanistic properties of archaeal-like membranes: their response to pressure, deformation and formation of the pores, as well as on more systematic assessment of membranes' permeability not only for water, but also for other solutes (e.g., ions) and gases. One should also note that due to the extraordinary properties of archaeal membranes, bolalipids will certainly provide some additional "degrees of freedom" in the construction of such materials. In other words, bolalipids significantly broaden the existing library of lipids used in development new membrane systems. These may be artificial membranes and vesicles with increased durability and carrying capacity, as well as unique surface properties that would define interaction with other agents, such as peptides and proteins.

\section{Computational Methods}

To study the effect of the branching groups of hydrophobic tails' on bolalipid membranes' properties, five mimetics of archaeal tetraether tail-linked bolalipids (GDGT) were chosen. The length of the main chain of the tail was 32 carbon atoms; the tails were linked by ether bonds to an archaeal-type glycerol backbone. To bring into focus the role of the hydrophobic core's chemical structure, polar heads were chosen to be just phosphatidylcholine (PC). The mimetics differed by the number and type of modifications in the main chain of the hydrophobic tail, from a complete absence of branching methyl groups $(\mathrm{m})$ and cyclopentane rings $(\mathrm{r})$ (this system is referred to as m0r0) to the introduction of eight modifications per tail in the m8r0 (eight methyls) and m6r2 (six methyls and two cyclopentane rings) systems. In addition, there were two intermediate variants with either two rings (m0r2) or two methyls ( $\mathrm{m} 2 \mathrm{r} 0)$. The position and chirality of the methyl groups and rings were taken from the structure of natural lipids. A summary of these modifications in the examined mimetics is provided in Fig. 1. As a reference, dipalmitoylphosphatidylcholine (DPPC) and palmitoyloleylphosphatidylcholine (POPC) were included in the study (also shown in Fig. 1).

Molecular dynamics (MD) was performed with Gromacs $4.5^{29}$ using a Slipids ${ }^{30}$ force field. This forcefield has been previously applied for detailed analysis of the behavior of different lipid systems at different temperatures, properties of the DPPC gel phase, multi component bilayers and protein-lipid interactions and demonstrated a good agreement with experimental data ${ }^{30,31}$. Lipid parameters provided by Slipids are compatible with Amber forcefield which permits their further application in studies of complex systems. Bolalipids topologies were created by analogy with "common" lipids, assuming all tail atoms were non-charged. For each system, four temperatures $(290,310,330$ and $350 \mathrm{~K})$ were examined; a Nose-Hoover thermostat was used. We didn't come up with higher temperatures (e.g. $370 \mathrm{~K}$ ) because this requires additional research on correctness of use of the forcefield, barostat and the water model for such high temperatures. Pressure (1 atm) was controlled in a semiisotropic manner with a Parrinello-Rahman barostat. The MD integration time was $0.002 \mathrm{ps}$; van der Waals interactions were truncated using the twin range 1.0/1.4 nm spherical cut-off. Electrostatic effects were treated using PME scheme. The length of each trajectory was 500 ns for bolalipids and 25 ns for phospholipids.

The examined systems contained 144 lipid and some 30000 tip 3 p water ${ }^{32}$ molecules (or 200 lipid molecules for DPPC and POPC). The starting configuration was created by translating a single lipid to a $12 \times 12$ grid with a step of $1 \mathrm{~nm}$ (yielding a $12 \times 12 \times$
$10 \mathrm{~nm}^{3} \mathrm{MD}$ box). Next, the water was added (with bulk density $1000 \mathrm{~kg} / \mathrm{m}^{3}$ ) and subsequently removed from the hydrophobic core area. Equilibration was performed in three steps: 1) 50 ps of MD with fixed volume (to get rid of system construction defects); 2) 250 ps of MD with a semi-isotropic volume coupling scheme using a Berendsen barostat and 3) 500 ps of MD with a semi-isotropic volume coupling scheme using a Parrinello-Rahman barostat for a better lateral adjustment of the lipid packing. To reduce the computational error, a 0.001 ps integration time step was used during equilibration. Thereafter, production MD runs were performed with a 0.002 ps integration time step.

To assess the lipid packing and phase condition of the model membranes, each bolalipid was represented as a vector built with two sn-2 glycerol carbon atoms from each polar head (Fig. 2A). The average lipid tilt angle $\chi$, average lipid-lipid collinearity angle $\varphi$ and average lipid "length" L (Fig. 2A; Fig. 3A-C) were calculated with inhouse scripts to assess packing characteristics. Partial density profiles (Fig. 3D) were calculated with a g_density program from the Gromacs suite. From these profiles, membrane thickness (as the distance between two phosphorus peaks) and hydrophobic core thickness (as a 99\%-drop of solvent density) were calculated (see Table S1).

As a measure of the "liquidity" of the hydrophobic slab, a root-mean-square fluctuation (RMSF) was used (see Fig. 4). In order to allow for an accurate comparison with phospholipids, the last $25 \mathrm{~ns}$ of each trajectory were used for the analysis. Analogous parameters were derived for DPPC and POPC bilayers, treating each chain as a vector similarly to bolalipids (Table S1). The identification of the longrange ordering in the model membranes (Fig. S4) was performed by implementing circular radial distribution functions (RDFs). First, a distance matrix for bolalipids centers of mass projections to the membrane plane was calculated. Second, the probability of finding a lipid within a certain distance from an arbitrarily chosen lipid was calculated $\left(\mathrm{P}(\mathrm{x}) ; \mathrm{R}_{\max }=3 \mathrm{~nm} ; \mathrm{dx}=0.002 \mathrm{~nm}\right)$. Third, the probability was normalized by the area of the ring with radius $\mathrm{x}$ and width $\mathrm{dx}: \mathrm{H}(\mathrm{x})=\mathrm{P}(\mathrm{x}) /(2 \pi \cdot \mathrm{x} \cdot \mathrm{dx})$. Finally, the RDFs were normalized by the area under the curve within a $0-3 \mathrm{~nm}$ range.

The permeability of the model membranes to water was assessed by calculating the relative frequency with which water molecules passed through the membrane ${ }^{26}$. A passage event was recorded if the water molecule crossed the membrane from one water volume to another (the border of the bulk water was defined by a distance of $3.7 \mathrm{~nm}$ from the middle plane of the MD box). The time step and analyzed trajectory length were $5 \mathrm{ps} / 50 \mathrm{~ns}$ for the DPPC/POPC bilayers and $10 \mathrm{ps} / 100 \mathrm{~ns}$ for the bolalipid mimetics membranes, respectively. The resulting frequency was normalized by the timescale and effective membrane area to make the comparison more straightforward (see Fig. 5 and Table S1).

The profiles of free volume along the $\mathrm{Z}$ coordinate (Figs. 6 and S5) and the distributions of void sizes and lifetimes were calculated as follows. The whole lipid (bi)layer was enclosed inside a box with dimensions $\mathrm{X} \times \mathrm{Y} \times 5 \mathrm{~nm}^{3}$, where $\mathrm{X}$ and $\mathrm{Y}$ are the sizes of the system. This box then became the source for a $150 \times 150 \times 50$ grid where each node was classified as either occupied with an atom (if it lied within the Van der Waals radius plus the radius of the water sphere, $0.14 \mathrm{~nm}$, from any atom) or void (Fig. S6). The analyzed trajectory length was $2 \mathrm{~ns}$; the number of consequent frames analyzed was 200 . Next, this four-dimensional grid was subjected to a $4 \mathrm{D}$ connected component labeling (4D-CCL) analysis, where each cluster of void nodes was numbered. This analysis yielded the lifetime of the given cluster along with the average cluster size (the sum of the instantaneous values divided by the number of frames). The profiles of the free volume were calculated as the percentage of void nodes in a given membrane XY-slab, depending on the Z-coordinate of the slab. These values were time-averaged and plotted along with standard deviations (Fig. 6). Other details of analysis of water moves and lipid clustering are given in refs. 26, 28, respectively.

1. Offre, P., Spang, A. \& Schleper, C. Archaea in biogeochemical cycles. Annu Rev Microbiol 67, 437-57 (2013).

2. Goldenfeld, N. Looking in the right direction: Carl Woese and evolutionary biology. RNA Biol 11 (2014).

3. Woese, C. R., Kandler, O. \& Wheelis, M. L. Towards a natural system of organisms: proposal for the domains Archaea, Bacteria, and Eucarya. Proc Natl Acad Sci U S A 87, 4576-9 (1990).

4. Koga, Y. From promiscuity to the lipid divide: on the evolution of distinct membranes in Archaea and Bacteria. J Mol Evol 78, 234-42 (2014).

5. Oger, P. M. \& Cario, A. Adaptation of the membrane in Archaea. Biophys Chem 183, 42-56 (2013).

6. Lombard, J., Lopez-Garcia, P. \& Moreira, D. Phylogenomic investigation of phospholipid synthesis in archaea. Archaea 2012, 630910 (2012).

7. Chong, P. L., Ayesa, U., Daswani, V. P. \& Hur, E. C. On physical properties of tetraether lipid membranes: effects of cyclopentane rings. Archaea 2012, 138439 (2012).

8. Chong, P. L., Sulc, M. \& Winter, R. Compressibilities and volume fluctuations of archaeal tetraether liposomes. Biophys J 99, 3319-26 (2010).

9. Bartucci, R., Gambacorta, A., Gliozzi, A., Marsh, D. \& Sportelli, L. Bipolar tetraether lipids: chain flexibility and membrane polarity gradients from spinlabel electron spin resonance. Biochemistry 44, 15017-23 (2005).

10. Chong, P. L., Ravindra, R., Khurana, M., English, V. \& Winter, R. Pressure perturbation and differential scanning calorimetric studies of bipolar tetraether 
liposomes derived from the thermoacidophilic archaeon Sulfolobus acidocaldarius. Biophys J 89, 1841-9 (2005).

11. Elferink, M. G., de Wit, J. G., Driessen, A. J. \& Konings, W. N. Stability and protonpermeability of liposomes composed of archaeal tetraether lipids. Biochim Biophys Acta 1193, 247-54 (1994).

12. Dante, S. et al. Thermal Stability of Bipolar Lipid Langmuir Blodgett Films by XRay Diffraction. Mol Cryst Liq Crys A 262, 191-207 (1995).

13. Chong, P. L. Archaebacterial bipolar tetraether lipids: Physico-chemical and membrane properties. Chem Phys Lipids 163, 253-65 (2010).

14. Boyd, E. S., Hamilton, T. L., Wang, J., He, L. \& Zhang, C. L. The role of tetraether lipid composition in the adaptation of thermophilic archaea to acidity. Front Microbiol 4, 62 (2013).

15. Zhai, Y. et al. Physical properties of archaeal tetraether lipid membranes as revealed by differential scanning and pressure perturbation calorimetry, molecular acoustics, and neutron reflectometry: effects of pressure and cell growth temperature. Langmuir 28, 5211-7 (2012).

16. Nishihara, M. \& Koga, Y. Extraction and composition of polar lipids from the archaebacterium, Methanobacterium thermoautotrophicum: effective extraction of tetraether lipids by an acidified solvent. J Biochem 101, 997-1005 (1987).

17. Dannenmuller, O. et al. Membrane properties of archaeal macrocyclic diether phospholipids. Chemistry 6, 645-54 (2000).

18. Stewart, L. C., Kates, M., Ekiel, I. H. \& Smith, I. C. P. Molecular order and dynamics of diphytanylglycerol phospholipids: a $2 \mathrm{H}$ and 31P-NMR study. Chem Phys Lipids 54, 115-129 (1990).

19. Benvegnu, T., Lemiègre, L. \& Cammas-Marion, S. Archaeal Lipids: Innovative Materials for Biotechnological Applications. Eur J Org Chem 2008, 4725-4744 (2008).

20. Bulacu, M., Periole, X. \& Marrink, S. J. In silico design of robust bolalipid membranes. Biomacromolecules 13, 196-205 (2012).

21. Gabriel, J. L. \& Chong, P. L. Molecular modeling of archaebacterial bipolar tetraether lipid membranes. Chem Phys Lipids 105, 193-200 (2000).

22. Shinoda, W., Shinoda, K., Baba, T. \& Mikami, M. Molecular dynamics study of bipolar tetraether lipid membranes. Biophys J 89, 3195-202 (2005).

23. Polyansky, A. A., Volynsky, P. E., Arseniev, A. S. \& Efremov, R. G. Adaptation of a membrane-active peptide to heterogeneous environment. II. The role of mosaic nature of the membrane surface. J Phys Chem B 113, 1120-6 (2009).

24. Pyrkova, D. V. et al. Dynamic clustering of lipids in hydrated two-component membranes: results of computer modeling and putative biological impact. J Biomol Struct Dyn 31, 87-95 (2013).

25. Volynsky, P. E., Polyansky, A. A., Simakov, N. A., Arseniev, A. S. \& Efremov, R. G. Effect of lipid composition on the "membrane response" induced by a fusion peptide. Biochemistry 44, 14626-37 (2005).

26. Krylov, N. A., Pentkovsky, V. M. \& Efremov, R. G. Nontrivial behavior of water in the vicinity and inside lipid bilayers as probed by molecular dynamics simulations. ACS Nano 7, 9428-42 (2013).

27. Franck, J. M., Scott, J. A. \& Han, S. Nonlinear scaling of surface water diffusion with bulk water viscosity of crowded solutions. J Am Chem Soc 135, 4175-8 (2013).

28. Pyrkova, D. V., Tarasova, N. K., Pyrkov, T. V., Krylov, N. A. \& Efremov, R. G. Atomic-scale lateral heterogeneity and dynamics of two-component lipid bilayers composed of saturated and unsaturated phosphatidylcholines. Soft Matter 7, 2569 (2011).

29. Hess, B., Kutzner, C., van der Spoel, D. \& Lindahl, E. GROMACS 4: Algorithms for Highly Efficient, Load-Balanced, and Scalable Molecular Simulation. J. Chem. Theory Comput. 4, 435-447 (2008).

30. Jambeck, J. P. \& Lyubartsev, A. P. Derivation and systematic validation of a refined all-atom force field for phosphatidylcholine lipids. J Phys Chem B 116, 3164-79 (2012).

31. Jämbeck, J. P. M. \& Lyubartsev, A. P. An Extension and Further Validation of an All-Atomistic Force Field for Biological Membranes. J Chem Theory Comput 8, 2938-2948 (2012).

32. Jorgensen, W. L., Chandrasekhar, J., Madura, J. D., Impey, R. W. \& Klein, M. L. Comparison of simple potential functions for simulating liquid water. J Chem Phys 79, 926 (1983).

\section{Acknowledgments}

A.O.C., I.A.B. and P.E.V. express their gratitude to the Russian Foundation for Basic Research (grants 13-04-40326-H and 13-04-00825) for support. This work was supported by Russian Scientific Foundation (grant 14-14-00871 awarded to R.G.E.). A.O.C. is recipient of the Stipend of the President of the Russian Federation. Authors also thank RAS Programmes "Basic fundamental research for nanotechnologies and nanomaterials" and "Molecular and Cellular Biology". Access to computational facilities of the Joint supercomputer center of RAS (Moscow) is greatly appreciated. We thank Ms. Anastasia Efremova for editing the manuscript.

\section{Author contributions}

A.O.C. performed data analysis and wrote the paper. P.E.V. set up and executed MD simulations. N.A.K. developed original analysis algorithms. I.A.B. proposed the experiment and prompted discussion. R.G.E. supervised the research and wrote the paper.

\section{Additional information}

Supplementary information accompanies this paper at http://www.nature.com/ scientificreports

Competing financial interests: The authors declare no competing financial interests.

How to cite this article: Chugunov, A.O., Volynsky, P.E., Krylov, N.A., Boldyrev, I.A. \& Efremov, R.G. Liquid but Durable: Molecular Dynamics Simulations Explain the Unique Properties of Archaeal-Like Membranes. Sci. Rep. 4, 7462; DOI:10.1038/srep07462 (2014).

This work is licensed under a Creative Commons Attribution-NonCommercialShareAlike 4.0 International License. The images or other third party material in this article are included in the article's Creative Commons license, unless indicated otherwise in the credit line; if the material is not included under the Creative Commons license, users will need to obtain permission from the license holder in order to reproduce the material. To view a copy of this license, visit http:// creativecommons.org/licenses/by-nc-sa/4.0/ 